\title{
Optimization of the Excitation signal for a novel piezoelectric micro-jet
}

\author{
Kai Li ${ }^{1, a}$, Jun-kao Liu ${ }^{1}$, Wei-shan Chen ${ }^{1}$ and Lu Zhang ${ }^{2}$ \\ ${ }^{1}$ State Key Laboratory of Robotics and System, Harbin Institute of Technology, Harbin 150001, China \\ ${ }^{2}$ AVIC, Harbin Dongan Engine Corporation LTD, Harbin 150001, China
}

\begin{abstract}
Fourier series expansions of different excitation signals for a novel piezoelectric micro-jet is deducted in this paper. By comparison and analysis of the Fourier series expansions, we find that the motivational effect of square wave is preferable than the sine wave which is better than triangle wave. And by analysing the Fourier series expansion of trapezoidal wave, it indicates that the ratio of the waveform parameters to the period should be studied rather than the numerical value of the parameters for improving the injection performance. The numerical simulations and experiments are carried out, and the results are consistent with theoretical calculations.
\end{abstract}

\section{Introduction}

The piezoelectric micro-jet, which can achieve drop-on-demand injection, is wildly used in various industrial fields [1-4]. The trapezoidal pulse wave is usually chosen as the excitation pulse waveform [5-7], however, there is no research results explaining the reasons for not choosing a triangular wave or sine wave instead. People mainly focuses on analysing the influences of trapezoidal wave parameters on the injection performance through experiments and simulations [8-11], or researching on the new method for controlling of the trapezoidal wave to reduce the residual vibration by using the self-sensing capability of the piezoelectric vibrator [12-14].

In order to obtain the optimal excitation signal which is applied on the piezoelectric micro-jet for achieving better injection performance, several typical waveforms are analysed depend on theoretical derivation in this paper. By analysing the derived results, the preferable excitation signal is given. Then, the analysis results are verified by simulations and experiments.

\section{Theoretical analysis}

The typical excitation waveform for piezoelectric micro-jet mainly include sine wave, triangle wave, square wave and trapezoidal wave. It's hard to find out which is preferred just by comparing the waveform functions or the shapes of the wave. As all functions (periodic function satisfies the Dirichelet conditions) can be expanded into a form of trigonometric functions by Fourier series expansion theory, the preferable waveform for better injection can be obtained by analysing the Fourier series expansions of different waveforms with the same voltage amplitude $\left(V_{m}\right)$ and period $(T)$.

The sine wave is shown in Fig.1, and its function expression with time as the variable is shown in Eq.(1).

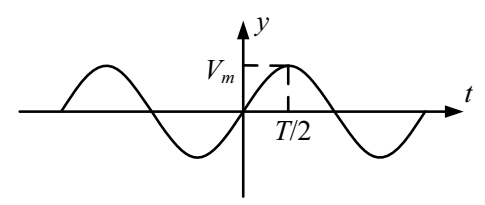

Figure 1. Function expression of sine wave.

$$
f_{\text {sine }}(t)=V_{m} \sin \left(\frac{2 \pi}{T} t\right)
$$

The triangle wave is shown in Fig.2, and its Fourier series expansion with time as the variable is shown in Eq.(2).

\footnotetext{
$\overline{{ }^{a} \text { Corresponding author: sdcxlikai@ } 126 . c o m}$
} 


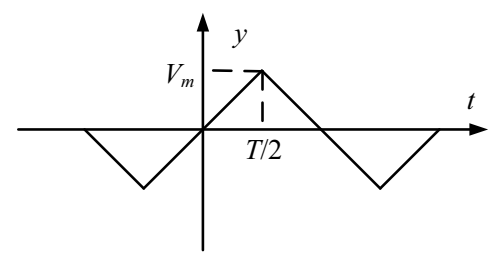

Figure 2. Fourier series expansion of triangle wave.

$$
f_{\text {triangle }}(t)=\frac{8}{\pi^{2}} V_{m} \sum_{n=1}^{\infty}\left[\frac{1}{n^{2}} \sin \left(\frac{n \pi}{2}\right) \sin \left(\frac{2 n \pi}{T} t\right)\right]
$$

The square wave is shown in Fig.3, and its Fourier series expansion with time as the variable is shown in Eq.(3).

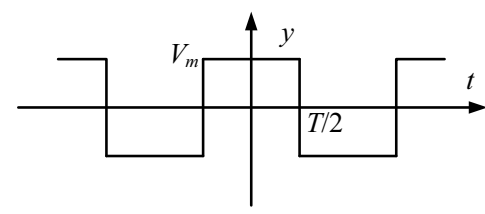

Figure 3. Fourier series expansion of square wave.

$$
f_{\text {square }}(t)=\frac{4}{\pi} V_{m} \sum_{n=1}^{\infty}\left[\frac{1}{n} \sin \left(\frac{n \pi}{2}\right) \cos \left(\frac{2 n \pi}{T} t\right)\right]
$$

The trapezoidal wave $\left(T_{r}=T_{f}\right)$ is shown in Fig.4, and its Fourier series expansion with time as the variable is shown in Eq.(4), where $T_{r}$ is rising time, $T_{h}$ is holding time, $T_{f}$ is falling time and $T_{s}$ is spacing time, $T_{r}+T_{h}+T_{f}+T_{s}=T$.

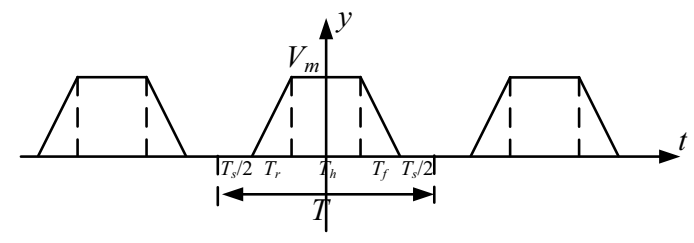

Figure 4. Fourier series expansion of trapezoidal wave $\left(T_{r}=T_{f}\right)$.

$$
\begin{aligned}
& f_{\text {trapezoidal }\left(T_{r}=T_{f}\right)}(t) \\
& =\frac{T_{h}+T_{f}}{T} V_{m}+\frac{T}{\pi^{2} T_{f}} V_{m} \sum_{n=1}^{\infty}\left[\frac { 1 } { n ^ { 2 } } \left(\cos \frac{T_{h}}{T} n \pi\right.\right. \\
& \left.\left.-\cos \frac{T_{h}+2 T_{f}}{T} n \pi\right) \cos \frac{2 n \pi}{T} t\right]
\end{aligned}
$$

The trapezoidal wave $\left(T_{r} \neq T_{f}\right)$ is shown in Fig.5, and its Fourier series expansion with time as the variable is shown in Eq.(5), where $a_{n}^{\prime}$ and $b_{n}^{\prime}$ are shown in appendix.

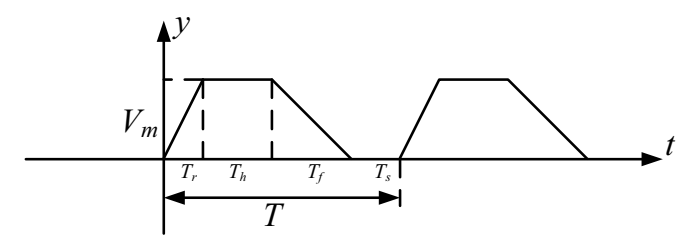

Figure 5. Fourier series expansion of trapezoidal wave $\left(T_{r} \neq T_{f}\right)$.

$$
\begin{aligned}
& f_{\text {trapezoidal }\left(T_{r} \neq T_{f}\right)}(t) \\
& =\frac{T_{r}+2 T_{h}+T_{f}}{2 T} V_{m} \\
& +\frac{T}{\pi^{2} T_{r} T_{f}} V_{m} \sum_{n=1}^{\infty} \frac{1}{n^{2}}\left(a_{n}^{\prime} \cos \frac{2 n \pi}{T} t+b_{n}^{\prime} \sin \frac{2 n \pi}{T} t\right)
\end{aligned}
$$

Thus, the fundamental components $(n=1)$ of these excitation signals are shown as follows:

$$
\begin{aligned}
& f_{\text {trinasle }}^{1}(t)=\frac{8}{\pi^{2}} V_{m} \sin \left(\frac{2 \pi}{T} t\right) \\
& f_{\text {square }}^{1}(t)=\frac{4}{\pi} V_{m} \cos \left(\frac{2 \pi}{T} t\right) \\
& f_{\text {trapecoida }\left(T_{r}=T_{f}\right)}^{1}(t) \\
& =\frac{T_{h}+T_{f}}{T} V_{m}+\frac{T}{\pi^{2} T_{f}} V_{m}\left[\cos \left(\frac{T_{h}}{T} \pi\right)\right. \\
& \left.-\cos \left(\frac{T_{h}+2 T_{f}}{T} \pi\right)\right] \cos \left(\frac{2 \pi}{T} t\right) \\
& f_{\text {trapeoxida }\left(T_{r} \neq f_{f}\right)}^{1}(t) \\
& =\frac{T_{r}+2 T_{h}+T_{f}}{2 T} V_{m}+\frac{T}{\pi^{2} T_{r} T_{f}} V_{m}\left[a_{n}^{\prime} \cos \frac{2 \pi}{T} t\right. \\
& \left.+b_{n}^{\prime} \sin \frac{2 \pi}{T} t\right]
\end{aligned}
$$

By comparing Eq.(1), (6), (7), (8) and (9), it demonstrate that the motivational effect of square wave is preferable than the sine wave which is better than triangle wave, as $8 / \pi^{2}<1<4 / \pi$ and the greater the excitation voltage amplitude, the better the performance of the injection. Meanwhile, due to the complex expressions of the Fourier series expansion of the trapezoidal wave, it is not easy to be compared with other waveforms. However, the square wave can be treated as a special form of the trapezoidal $\left(T_{r}=T_{f}=0\right)$, the trapezoidal wave may be more effective as well than other waveforms. And by analysing Eq.(8) and (9), it indicates that the ratio of the waveform parameters to the period should be 
studied rather than the numerical value of the parameters for improving the injection performance. In order to prove the correction of the analysis above, numerical analysis of a novel piezoelectric micro-jet is carried out next.

\section{Numerical analysis}

In this paper, we use the novel piezoelectric micro-jet which is designed for bearing lubrication by our group, for more details, please refer to the references[8].The structure of the novel piezoelectric micro-jet is shown in Fig.6, and all the simulation parameter settings are in agreement with the references[8].

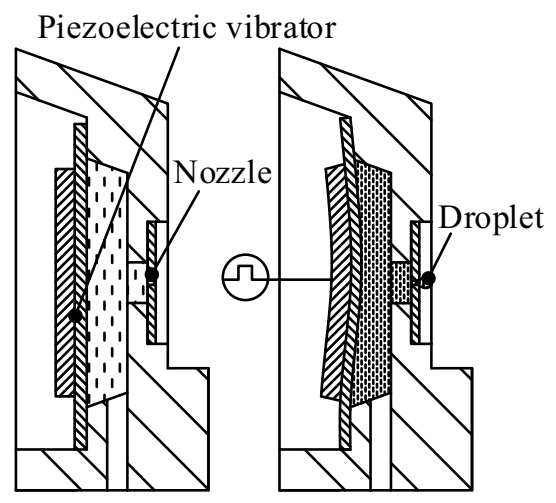

Figure 6. The structure of the micro-jet.[8]

We select the sine wave, trapezoidal wave $\left(T_{r}: T_{h}: T_{f}=1: 1: 1\right)$, triangular wave and square wave as the excitation signals with the same voltage amplitude $\left(V_{m}=100 \mathrm{~V}\right)$ and period ( $T=55.77 \mathrm{us}$ ). The displacement and velocity of one of the particles near the nozzle are studied by a finite element analysis. The curves of the vibration displacement of the same particle with different excitation signals is shown in Fig.7.

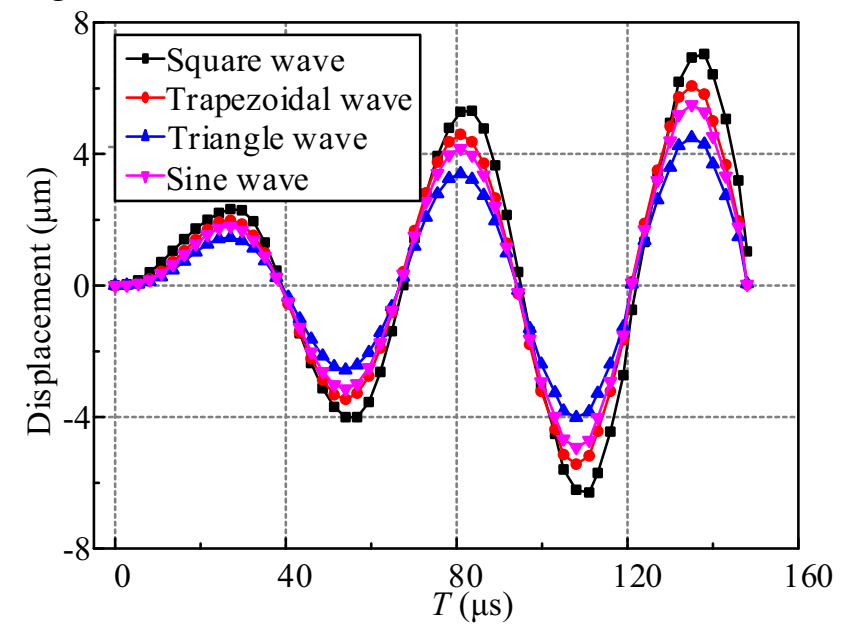

Figure 7 The he vibration displacement of the same particle with different excitation signals.

As we can see that the vibration displacement of the particle under the excitation of square wave is larger than that of trapezoidal wave or sine wave which are greater than that of triangle wave. This is consistent with the theoretical calculation. The curves of the vibration velocity of the same particle with different excitation signals is shown in Fig.8.

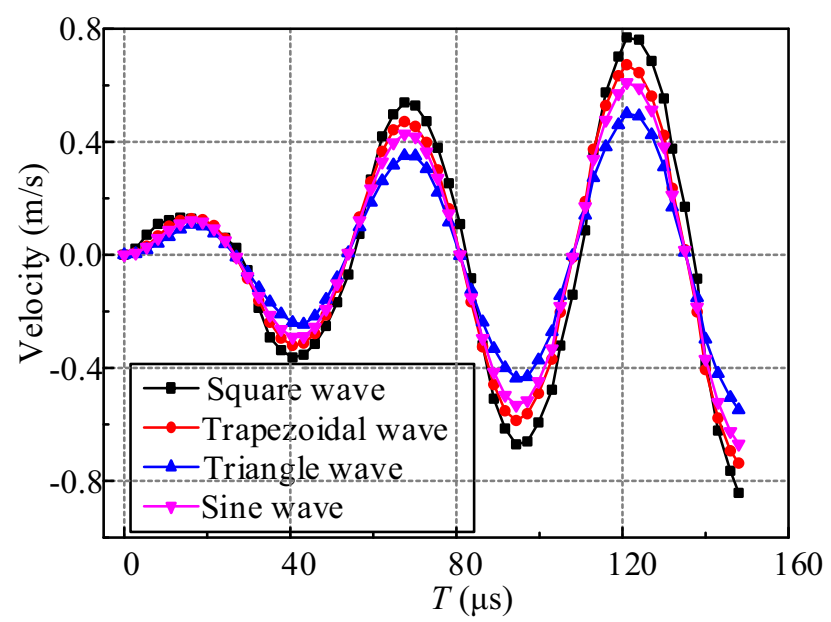

Figure 8. The he vibration displacement of the same particle with different excitation signals.

It can be seen that when the piezoelectric micro-jet is applied different excitation signals with the same voltage amplitude $\left(V_{m}=100 \mathrm{~V}\right)$ and period ( $T=55.77 \mathrm{us}$ ), the velocity of the particle under the excitation of triangle wave is less than that of trapezoidal wave or sin wave which are inferior to that of square wave. This is also consistent with the theoretical calculation. The theoretical and numerical simulation results are verified by experiments, as described below.

\section{Experimental analysis}

An experimental research is carried out by using the experimental method as shown in references[8], and the properties of injection lubricating oil and the experimental equipment are the same as what is described in references[8]. In order to show the influences of the excitation signals obviously, only the square wave which obtain the best incentive effect and the sine wave which obtain the worst effect are selected in the experiments. 
The injection status of the micro-jet under different excitation signals with same voltage amplitudes and period, are shown in Fig.9.
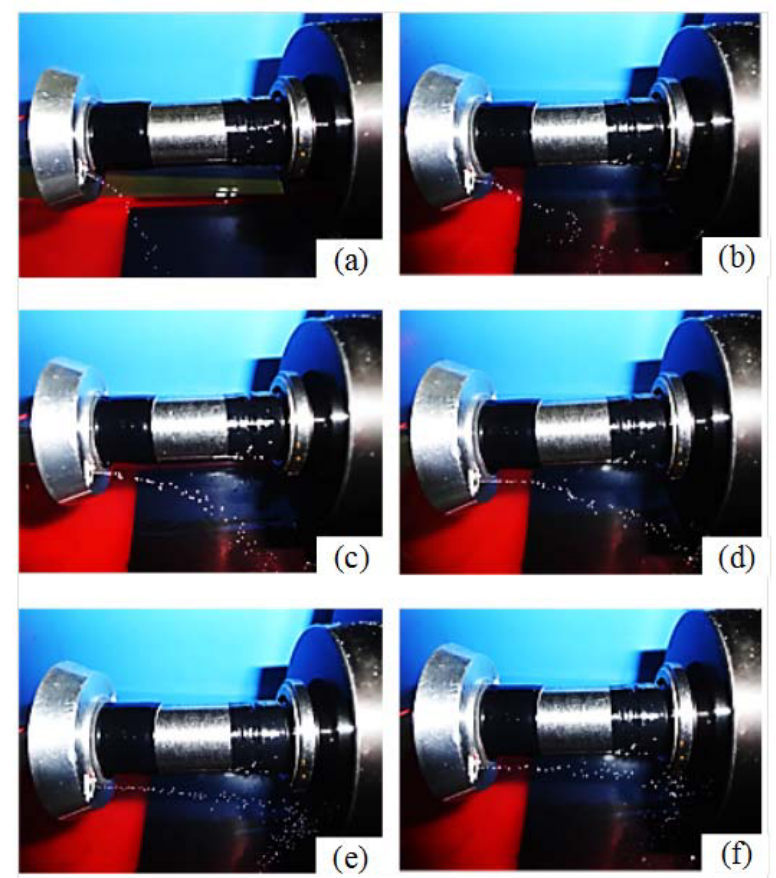

Figure 9. Injection status of the micro-jet under different excitation signals :(a) sine wave, and $V_{m}=60 \mathrm{~V}$; (b) square wave, and $V_{m}=60 \mathrm{~V}$; (c) sine wave, and $V_{m}=80 \mathrm{~V}$; (d) square wave, and $V_{m}=80 \mathrm{~V}$; (e) sine wave, and $V_{m}=100 \mathrm{~V}$; (f) square wave, and $V_{m}=100 \mathrm{~V}$.

As we can see from Fig.9, whether the excitation signal is sine wave or square wave, the injection intensity of the micro-jet increases along with the increase of the voltage amplitude. Meanwhile, the injection status of the micro-jet under the excitation of square wave is more intense than that of sine wave when the voltage amplitude of the excitation signal keeps the same. The experimental results are in agreement with the simulations and theoretical calculations.

Another evaluation of the piezoelectric micro-jet is the mass of the droplet, the mass of the droplet varies with different voltage amplitude of excitation signals are shown in Fig.10. It can be seen that the droplet mass increase gradually with the increase of the voltage amplitude for each excitation signals. And the mass of the droplet ejected out of the micro-jet under the square wave is larger than that of sine wave when the voltage amplitude keep the same. This also verifies the correctness of simulations and theoretical calculations.

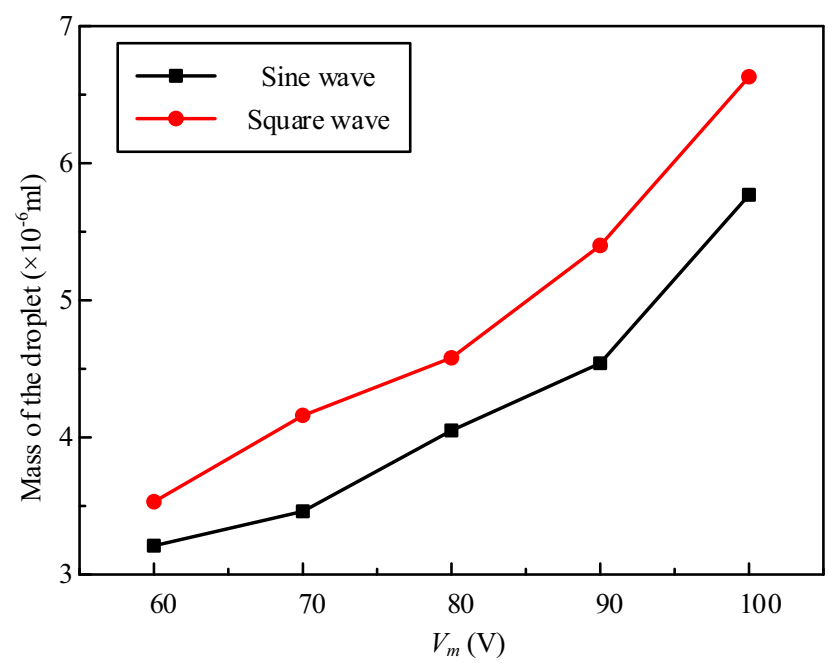

Figure 10. The change curves of the droplet mass along with the voltage amplitude of excitation signals.

\section{Conclusions}

A new method for analysing the excitation signals of the piezoelectric micro-jet is proposed in this paper. The theoretical calculations prove that the square wave or trapezoidal wave are more effectively than sine wave or square wave by the discussions of the Fourier series expansions of excitation signals. Simulations and experiments are carried out to verify the correctness of the theoretical calculations, and the results are in agreement with the theoretical calculations. Meanwhile, the ratio of the waveform parameters of the trapezoidal wave to the period should be studied rather than the numerical value of the parameters for improving the injection performance according to the analysis of the Fourier series expansion of the trapezoidal wave. 


\section{Appendix}

$$
\begin{aligned}
a_{n}^{\prime}= & T_{f} \cos ^{2}\left(\frac{T_{r}}{T} n \pi\right)-2 T_{r} \cos ^{2}\left(\frac{T_{r}}{T} n \pi\right)-2 T_{r} \cos ^{2}\left(\frac{T_{h}}{T} n \pi\right) \\
& -T_{r} \cos ^{2}\left(\frac{T_{f}}{T} n \pi\right) \\
& +4 T_{r} \cos ^{2}\left(\frac{T_{r}}{T} n \pi\right) \cos ^{2}\left(\frac{T_{h}}{T} n \pi\right) \sin ^{2}\left(\frac{T_{f}}{T} n \pi\right) \\
& +2 T_{r} \cos ^{2}\left(\frac{T_{r}}{T} n \pi\right) \cos ^{2}\left(\frac{T_{f}}{T} n \pi\right) \\
& +2 T_{r} \cos ^{2}\left(\frac{T_{h}}{T} n \pi\right) \cos ^{2}\left(\frac{T_{f}}{T} n \pi\right) \\
& -\frac{1}{2} T_{r} \sin \left(\frac{2 T_{r}}{T} n \pi\right) \sin \left(\frac{2 T_{f}}{T} n \pi\right) \\
& -T_{r} \sin \left(\frac{2 T_{r}}{T} n \pi\right) \sin \left(\frac{2 T_{h}}{T} n \pi\right) \\
& -\frac{1}{2} T_{r} \sin \left(\frac{2 T_{h}}{T} n \pi\right) \sin \left(\frac{2 T_{f}}{T} n \pi\right) \\
& +T_{r} \sin \left(\frac{2 T_{r}}{T} n \pi\right) \sin \left(\frac{2 T_{h}}{T} n \pi\right) \cos ^{2}\left(\frac{T_{f}}{T} n \pi\right) \\
& +T_{r} \sin \left(\frac{2 T_{h}}{T} n \pi\right) \sin \left(\frac{2 T_{f}}{T} n \pi\right) \cos ^{2}\left(\frac{T_{r}}{T} n \pi\right) \\
& +T_{r} \sin \left(\frac{2 T_{r}}{T} n \pi\right) \sin \left(\frac{2 T_{f}}{T} n \pi\right) \cos ^{2}\left(\frac{T_{h}}{T} n \pi\right)+T_{r}-T_{f} \\
&
\end{aligned}
$$$$
b_{n}^{\prime}=2 T_{r} \sin \left(\frac{2 T_{r}}{T} n \pi\right) \cos ^{2}\left(\frac{T_{h}}{T} n \pi\right)
$$$$
+2 T_{r} \sin \left(\frac{2 T_{h}}{T} n \pi\right) \cos ^{2}\left(\frac{T_{r}}{T} n \pi\right)
$$$$
+T_{r} \sin \left(\frac{2 T_{r}}{T} n \pi\right) \cos ^{2}\left(\frac{T_{f}}{T} n \pi\right)
$$$$
+T_{r} \sin \left(\frac{2 T_{f}}{T} n \pi\right) \cos ^{2}\left(\frac{T_{r}}{T} n \pi\right)
$$$$
+T_{r} \sin \left(\frac{2 T_{h}}{T} n \pi\right) \cos ^{2}\left(\frac{T_{f}}{T} n \pi\right)
$$$$
+T_{r} \sin \left(\frac{2 T_{f}}{T} n \pi\right) \cos ^{2}\left(\frac{T_{h}}{T} n \pi\right)
$$$$
-2 T_{r} \sin \left(\frac{2 T_{r}}{T} n \pi\right) \cos ^{2}\left(\frac{T_{h}}{T} n \pi\right) \cos ^{2}\left(\frac{T_{f}}{T} n \pi\right)
$$$$
-2 T_{r} \sin \left(\frac{2 T_{h}}{T} n \pi\right) \cos ^{2}\left(\frac{T_{r}}{T} n \pi\right) \cos ^{2}\left(\frac{T_{f}}{T} n \pi\right)
$$$$
-2 T_{r} \sin \left(\frac{2 T_{f}}{T} n \pi\right) \cos ^{2}\left(\frac{T_{r}}{T} n \pi\right) \cos ^{2}\left(\frac{T_{h}}{T} n \pi\right)
$$$$
+\frac{1}{2} T_{f} \sin \left(\frac{2 T_{r}}{T} n \pi\right)-T_{r} \sin \left(\frac{2 T_{r}}{T} n \pi\right)
$$$$
-T_{r} \sin \left(\frac{2 T_{h}}{T} n \pi\right)-\frac{1}{2} T_{r} \sin \left(\frac{2 T_{f}}{T} n \pi\right)
$$$$
+\frac{1}{2} T_{r} \sin \left(\frac{2 T_{r}}{T} n \pi\right) \sin \left(\frac{2 T_{h}}{T} n \pi\right) \sin \left(\frac{2 T_{f}}{T} n \pi\right)
$$

\section{References}

1. M Alomari,F H Mohamed,A W Basit,S Gaisford. Int. J. Pharm. , 494 568-577 (2015)

2. J Xu,D Attinger. J. Micromech. Microeng., 1810 (2008)

3. I Van Driessche,J Feys,S C Hopkins,P Lommens, X Granados,B A Glowacki,S Ricart,B Holzapfel,M Vilardell,A Kirchner, et al. Superconductor Science \& Technology, 2512 (2012)

4. S Rahmati,S F Shirazi,H Baghayeri. Rapid Prototyping Journal, 15 187-191 (2009)

5. J Brunahl,A M Grishin. Sensors and Actuators aPhysical, 101 371-382 (2002)

6. M-H Tsai,W-S Hwang. Mater. Trans. , 49 331-338 (2008)

7. H J Lin, $\mathrm{H} \mathrm{C} \mathrm{Wu,T} \mathrm{R} \mathrm{Shan,W} \mathrm{S} \mathrm{Hwang.} \mathrm{Mater.}$ Trans. , 47 375-382 (2006)

8. K Li,J Liu,W Chen,L Ye,L Zhang. Applied Sciences, 638 (2016)

9. S Kim,J Sung,M H Lee. J. Therm. Sci. , 22 29-35 (2013)

10. T M Liou,C Y Chan,K C Shih. Microfluid. Nanofluid., 8 575-586 (2010)

11. W J Shin,Y S Jeong,K Choi,W G Shin. Aerosol Sci. Technol. , 49 1256-1262 (2015)

12. K-S Kwon,김우식. Transactions of the Korean Society for Noise and Vibration Engineering, 17 333-341 (2007)

13. A A Khalate, $X$ Bombois, $G$ Scorletti, R Babuska,S Koekebakker,W De Zeeuw. Journal of Microelectromechanical Systems, 21 1365-1374 (2012)

14. K S Kwon,W Kim. Sensors and Actuators aPhysical, 140 75-83 (2007) 